\title{
MAXIMUM DAMPING OF THE LOWEST ROOM EIGENFREQUENCY BY A MONOPOLE
}

\author{
Darya $\mathrm{M}$ ameshina ${ }^{{ }^{*}}$, and Nikolay $\mathrm{K}$ anev ${ }^{1,2}$ \\ ${ }^{1}$ B M STU, Power Engineering Department, 105005, M oscow, Russian Federation \\ ${ }^{2}$ A ndreyev A coustic Institute, 117036, M oscow, Russian Federation
}

\begin{abstract}
In this paper, we consider the problem of sound absorption by a monopole in a rectangular room with absolutely rigid walls. The absorption efficiency is characterized by the damping coefficients of free oscillations of the "monopole-room" system. The impedance of the monopole is found, at which the damping coefficient of the resonance of the system with the lowest natural frequency is maximum. The monopole with this impedance can be realized using an active control method that allows the required impedance to be achieved over a wide frequency band.
\end{abstract}

\section{Introduction}

Noise control is one of the main areas of modern acoustics. Acoustic resonators are an effective means of dealing with noise. It is sufficient to mention the resonant coatings of room walls and air ducts and resonant mufflers for narrow pipes and waveguides. The main element here is the Helmholtz resonator [1-8].

The advantage of resonators over alternative passive noise suppression devices is their small wave size. In free space, the maximum characteristic absorption and scattering cross sections of resonators depend on only one parameter which is the sound wavelength. Let us give only one well-known example [9]: the absorption cross section of a gas bubble in liquid is several hundred times its cross-sectional area. Since the efficiency of resonant sound control increases with decreasing wavelength, it is most effective to use resonators to control low-frequency noise.

Many small (compared to the sound wavelength) resonators, for example, a Helmholtz resonator or a gas bubble in a liquid, studied and proposed in modern literature, are of the monopole type, i.e. as sources of the scattered field, they are monopoles. A traditionally used resonator is an obstacle with a compressibility different from the compressibility of the medium, for which the resonance condition is satisfied: the equality of the imaginary part of the impedance to zero at a certain frequency.

For maximum absorption in the room, the resonator must be tuned to a certain frequency and have a certain friction coefficient [10]. In this case, the mass of the resonator should be as small as possible. Formally, one can direct the length of the resonator neck to zero and find the maximum possible attenuation coefficient for low-frequency room modes. However,

* Corresponding author: author@email.org 
in the limit of zero length, the resonator mass is nonzero - it is the added mass of the hole. Thus, the efficiency of the Helmholtz resonator has physical limitations [8,11].

To obtain a better result, the absorption of sound in a room by a monopole with an arbitrary impedance, independent of geometric parameters, is considered. The task is to determine the maximum possible absorption introduced by the monopole, as well as the value of its impedance at which it is achieved.

\section{Formulation of the problem}

Let us present the solution to the well-known problem of sound absorption by a Helmholtz resonator in a room at the lowest natural frequency. The sound field in a rectangular room with a Helmholtz resonator was found in the work of A.D. Lapin. [12]. According to this work, the resonant frequencies can be found from the equation

$$
Z(\omega)+Z_{r}(\omega)=0
$$

where $Z$ is the acoustic impedance of the resonator, $Z_{r}$ is the radiation resistance of the resonator in the room.

Impedances can be written as follows:

$$
\begin{aligned}
& Z=\frac{1}{s^{2}}\left(R+\frac{K}{-i \omega}-i \omega M\right) \\
& Z_{r}=\frac{i \omega \rho}{V} \sum_{n} \frac{p_{n}^{2}(r)}{k^{2}-k_{n}^{2}}
\end{aligned}
$$

where $S$ is the cross-sectional area of the resonator neck, $R$ is the friction coefficient, $K$ is the elasticity coefficient, $\omega$ is the sound frequency, $M$ is the air mass in the resonator neck, $\rho$ is the density of the medium, $V$ is the volume of the room, $k=\omega / c, k_{n}=\omega_{n} / c, p_{n}(r)$ are fundamental functions.

All frequencies are normalized to $\omega_{1}$ which is the first (i.e., the lowest) resonant frequency of the room in the absence of a resonator. Further, dimensionless frequencies are used

$$
\bar{\omega}=\frac{\omega}{\omega_{1}}, \overline{\omega_{0}}=\frac{\omega_{0}}{\omega_{1}}
$$

We introduce the following dimensionless quantities characterizing the resonator mass and friction coefficient

$$
\bar{R}=\frac{R}{\omega_{1} M}, m=\left(\frac{\rho c^{2} S^{2}}{V M \omega_{1}^{2}}\right)^{-1}
$$

According to the introduced notation, we transform (1) to an equation in the form of a polynomial and solve numerically

$$
\bar{\omega}^{4}(-m)+\bar{\omega}^{3}(-\bar{R} m i)+\bar{\omega}^{2}\left({\overline{\omega_{0}}}^{2} m+m+1\right)+\bar{\omega}(\bar{R} m i)-{\overline{\omega_{0}}}^{2} m=0
$$

When solving the problem of low-frequency absorption by a Helmholtz resonator in a room with absolutely rigid walls, the maximum achievable damping coefficient of the natural oscillations of the room was found [10]. It is shown that the parameter $m$ cannot take values less than 100, which causes a limitation of the damping coefficient. The minimum possible value of the parameter $m$ is related to the added mass of the resonator: even if the resonator 
has no neck, its mass is determined by the added mass of the hole. Nevertheless, in order to understand the effect of the resonator mass on the sound absorption, we will consider two cases: $m=100$ is the minimum possible resonator mass, $m=1$ is a physically unrealizable case of the small Helmholtz resonator mass.

Figure 1 shows the graphs for the case when the natural frequency of the resonator in free space coincides with the first natural frequency of the room without the resonator. When $\bar{R}=0$ (punctured points on the graph), there are two natural frequencies: $\Omega_{0}$ is the natural frequency of the resonator in the room, $\Omega_{1}$ is the natural frequency of the room with the resonator. Both frequencies lie on the real axis, i.e. the corresponding natural oscillations of the system occur without damping, since the resonator does not absorb sound energy. With an increase in the resonator friction $\bar{R}$ (the movement of the root with an increase in $\bar{R}$ is shown by an arrow), both roots (4) become complex, i.e. both modes become damped. To characterize the rate of damping of modes, we introduce damping coefficients $\delta_{0}=-\operatorname{Im} \Omega_{0}$ and $\delta_{1}=-\operatorname{Im} \Omega_{1}$. Gradually, the roots approach each other and for some value of $\mathrm{R}^{-}$take the same values, i.e. become multiple roots. In this case, the damping coefficients of both modes reach their maximum value. With a further increase in the friction coefficient $\bar{R}$, one of the roots becomes purely imaginary, and the other tends to 1 , i.e. to the first natural frequency of the room without a resonator.

Thus, the maximum absorption introduced by the Helmholtz resonator (Fig. 1a) provides the damping coefficient of the eigenmodes $\delta_{0}=\delta_{1}=0.05$. In this case, the friction coefficient is $\bar{R}=0,2$. If the cavity mass could be made arbitrarily small, then the damping coefficient could be an order of magnitude larger (Fig. 1b).

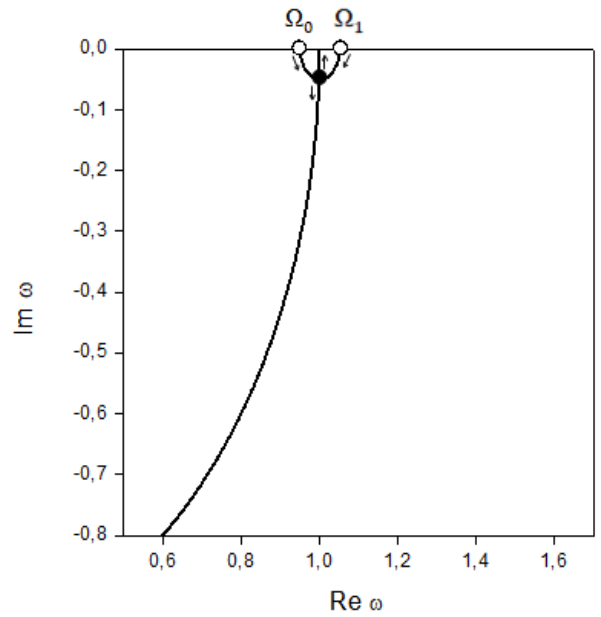

a

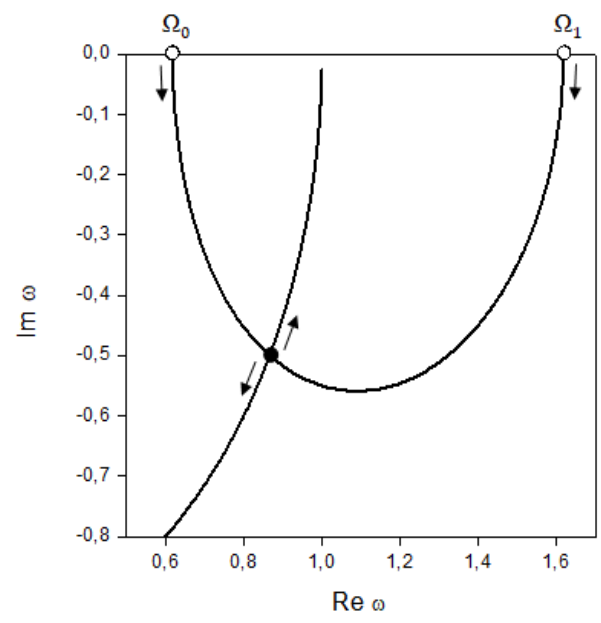

$\mathrm{b}$

Fig. 1. Graphs of the natural frequencies of the «room-resonator» system when the friction coefficient $\bar{R}$ changes from 0 to $\infty$ for the values $\overline{\omega_{0}}=1$ and $a$ ) $\left.m=100 ; b\right) m=1$.

When comparing the two graphs, one can clearly see how the damping coefficient changes as $m \rightarrow 0$. Obviously, the higher the value of the parameter $m$, the less the effect of the resonator on the natural frequencies of the room. However, taking into account the physical limitations of the Helmholtz resonator mass, the parameter $\mathrm{m}$ and, therefore, the impedance cannot take arbitrary values.

In this paper, it is proposed to investigate the efficiency of sound absorption by a monopole with arbitrary impedance in a rectangular room with absolutely rigid walls. 
Consider a monopole with impedance $Z$. We assume that it can take any value. Also assume that the monopole is located in the corner of the room, then $p_{n}^{2}(r)=1$. Thus, equation (1) can be written in the following form

$$
Z+\frac{i \omega \rho}{V} \sum_{n} \frac{1}{k^{2}-k_{n}^{2}}=0
$$

We replace the wave numbers $k=\omega / c$ and $k_{n}=\omega_{n} / c$. Then (5) is transformed to the form

$$
Z+\frac{i \omega \rho c^{2}}{V} \sum_{n} \frac{1}{\omega^{2}-\omega_{n}^{2}}=0
$$

Let us introduce dimensionless parameters of the problem. To do this, we divide (6) by $\frac{\rho c^{2}}{V \omega_{1}}$ and write in the following form

$$
\frac{Z}{\frac{\rho c^{2}}{V \omega_{1}}}+i \frac{\omega}{\omega_{1}} \sum_{n} \frac{1}{\left(\frac{\omega}{\omega_{1}}\right)^{2}-\left(\frac{\omega_{n}}{\omega_{1}}\right)^{2}}=0
$$

Let us introduce the following dimensionless quantities, keeping the previous designations.

The monopole impedance is denoted as

$$
\bar{Z}=\frac{Z}{\frac{\rho c^{2}}{V \omega_{1}}}
$$

The resonant frequencies of the «room-monopole» system and the frequency as

$$
\overline{\omega_{n}}=\frac{\omega_{n}}{\omega_{1}}, \bar{\omega}=\frac{\omega}{\omega_{1}}
$$

According to the notation in formulas (8) and (9), we transform (7) to the form

$$
\bar{Z}+i \bar{\omega}\left(\frac{1}{\bar{\omega}^{2}-1}+\sum_{n \geq 2} \frac{1}{\bar{\omega}^{2}-\bar{\omega}_{n}^{2}}\right)=0
$$

We introduce the coefficient $\alpha=\sum_{n \geq 2} \frac{1}{\bar{\omega}^{2}-{\overline{\omega_{n}}}^{2}}$, which can be considered constant in the vicinity of the first resonant frequency. We obtain the final equation for the natural frequencies of the «room-monopole» system

$$
\bar{Z}+i \bar{\omega}\left(\frac{1}{\bar{\omega}^{2}-1}+\alpha\right)=0
$$




\section{Eigenfrequencies of a room with a monopole}

Equation (11) can be solved numerically. For this, (11) must be reduced to the form of a polynomial

$$
\bar{\omega}^{3}(i \alpha)+\bar{\omega}^{2}(\bar{Z})+\bar{\omega}(1-\alpha) i-\bar{Z}=0
$$

Equation (12) is a third degree polynomial, so it has three roots. Two of them $\overline{\omega_{1}}$ and $\overline{\omega_{2}}$ the first resonant frequencies of the «room-monopole» system. If the real part of the impedance $\bar{Z}$ is not zero, then both roots $\overline{\omega_{1}}$ and $\overline{\omega_{2}}$ are complex, and their imaginary part determines the decay rate of the corresponding natural oscillation. To characterize the damping of oscillations in the vicinity of the first natural frequencies, we choose the minimum of the two damping coefficients

$$
\delta=\min \left(-\operatorname{Im} \overline{\omega_{1}},-\operatorname{Im} \overline{\omega_{2}}\right)
$$

It is obvious that the damping coefficient $\delta$ is a function of the impedance $\bar{Z}$. Figure 2 shows the dependence of this function in the form of lines of equal values of the attenuation coefficient in the plane $(\operatorname{Re} \bar{Z}, I \mathrm{~m} \bar{Z})$. For the calculation, the value of the coefficient $\alpha=1.26$ was taken, calculated for a room, the aspect ratio of which is 1:0.5: 0.3 [11].

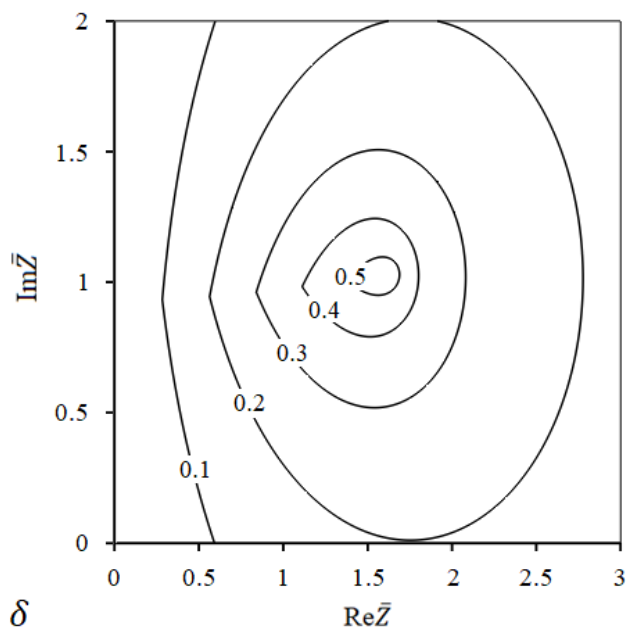

Fig. 2. Dependence of the oscillation damping coefficient $\delta$ on the monopole impedance $\bar{Z}$ in the form of lines of equal values.

This graph clearly shows that at some values of the monopole impedance $\bar{Z}$, the damping coefficient $\delta$ takes values that are several times higher than the maximum attainable damping coefficient of the natural vibration of the room when sound is absorbed by the Helmholtz resonator. And the maximum absorption introduced by the monopole provides the damping coefficient of the first modes $\delta=0.598$. It is achieved when sound is absorbed by the monopole with impedance, the real and imaginary parts of which are $\mathrm{Re} \bar{Z}=1.65$, $\operatorname{Im} \bar{Z}=1.032$.

Obviously, using a monopole with optimal impedance for absorbing sound in a room is more effective than using a Helmholtz resonator, since it allows the greater damping coefficient at a low resonant frequency. 


\section{Implementation of the optimum impedance}

Optimal impedance monopole can be implemented with an active control method that allows arbitrary monopole impedance over a wide frequency band. The essence of the method is to control the movement of the monopole depending on the sound field in its vicinity. At each frequency of a certain range in an active way, such an oscillatory speed of the monopole is selected so that it coincides with the corresponding speed required to implement the required impedance [13]. The control signal is generated from the sound pressure value measured in the vicinity of the monopole.

This approach actually optimizes the monopole impedance in a wide frequency band and, thus, can be attributed to one of the modern approaches in the field of active sound control which is the impedance matching technique [14-19]. The proposed method of active damping based on local control has a simple principle of use. If the solution to the problem of sound absorption by a monopole is known, then this solution is generalized to all frequencies of a given range. Thus, the problem is reduced to finding a control function (or algorithm), depending on the frequency, which ultimately sets the required oscillatory speed of the monopole.

Technically, an active absorber can be created using a loudspeaker, a microphone located close to its surface, and a frequency-dependent feedback filter that transmits the signal from the microphone to the loudspeaker. The feedback coefficient is selected in such a way as to provide the optimal monopole impedance (for maximum absorption at the first resonant frequency of the room $\bar{Z}=1.65+1.032 i$ in a given frequency band.

\section{Conclusion}

In this work, the problem of the maximum sound absorption by a monopole in a rectangular room with absolutely rigid walls is solved. The impedance of the monopole, at which the damping coefficient of the resonance of the system with the lowest natural frequency is maximum, is found, as well as its value. In this case, the damping coefficient, equal to about 0.5 , is an order of magnitude higher than the maximum damping coefficient provided by the Helmholtz resonator [2,11].

Monopole with the required impedance can be implemented by means of an active control method that allows the specified impedance to be achieved over a wide frequency band.

\section{References}

1. A. Selamet, N. S. Dicky, J. M. Novak, J. Sound V ib., 187, 358-367 (1995)

2. N. G. Kanev, A coust. Phys., 64(6), 774-777 (2018)

3. A. I. Komkin, M. A. M ironov, A. I. Bykov, A coust. Phys., 63(4), 385-392 (2017)

4. H. Zhongyu, C. Y ang., L. Cheng, A ppl. A coust., 134, 88-96 (2018)

5. A. I. Komkin, M. A. M ironov, S. I. Y udin, A coust. Phys., 60(2), 142-147 (2014)

6. N. Kanev, A coustics, 2(2), 399-415 (2020)

7. F. J. Fahy, C. A. Schofield, J. Sound V ib., 72, 365-378 (1980)

8. A . I. Komkin, A. I. Bykov, A coust. Phys., 62(3), 269-279 (2016)

9. M. A. Isakovich, General acoustics. Textbook, M oscow: Nauka, 363-364 (1973)

10. J. Klaus, I. B ork, M. Graf, G.-P. Ostermeyer, A ppl. A coust., 77, 37-41 (2014) 
11. D. A . M ameshina, N. G. K anev, Low-frequency absorption of sound by a Helmholtz resonator in a room, in Proceedings of $3^{\text {rd }}$ Conference of $Y$ oung Scientists and Specialists "A coustics of the environment", A SO-2018, 18 M ay 2018, M oscow (2018)

12. A. D. Lapin, A coust. Phys., 46(4), 491-493 (2000)

13. N. G. Kanev, M. A. M ironov, A coust. Phys., 54(3), 437-443 (2008)

14. C. H. Hansen, S. D. Snyder, Active control of noise and vibration, E\& FN, London, (1997)

15. Y u. I. Bobrovnitskiy, A coust. Phys., 49(6), 620-626 (2003)

16. N. Kanev, M. M ironov, A. Gladilin, Robustness of a feedback active noise control, Proc. of INTERN OISE 2010, 13-16 J une 2010, Lisbon, Portugal (2010)

17. R. B oulandet, E. Rivet, H. Lissek, A cta A custica united with A custica, 102, 696-704 (2016)

18. N. K anev, Adjustability of acoustic properties of surfaces at low frequencies by an array of active resonators, Proc. of Euronoise 2018 Conference, 28-31 M ay 2018, Crete, Greece (2018)

19. V. M artin, A. Bodrero, J. Sound. V ib., 204, 331-357 (1997) 